Historic, Archive Document

Do not assume content reflects current scientific knowledge, policies, or practices. 



\section{WHOLESALE - PRICE - LIST FALL 1925 - SPRING 1926}

Liardwood cuttings, Seed, Seedlings, and lining out stock.

\section{Southern Grown Cuttings Best}

Ask any florist which roots the best, and propagates the best and rrows off most freely-CUTTINGS from outdoor wood or from indoor wood, (wood grown under glass). Ninety nine per cent will answer, "Indoor wood." WHY IS THIS TRUE? The indoor wood is more porus, is not as hard grained, and, therefore callouses and throws out roots more readily than the hard grained outdoor wood.

The outdoor wood is likely to make big hard callouses with no roots, while the indoor wood makes SOFT callouses which throw out healthy young roots, and the plants start off freely, and grow vigorously.

In the same way and for the same reason, SOUTHERN grown Cutlings root more FREELY, and start off more vigorously than Northern grown cuttings.

Ask any florist, or try a few southern grown cuttings and be convinced. We make a specialty of southern grown cuttings.

Hardwood cuttings should be fresh, with all their vitality in them. For this reason we try to ship our cuttings as fast as they are made up.

The enclosed list of QUALITY CUTTTINGS will give you good results if you place your order with us.

ROSENBANK NURSERY CO., Inc.

Huntsville, Alabama.

\section{HARDWOOD CUTTINGS}

\begin{tabular}{|c|c|c|}
\hline & Per & Per \\
\hline river, Amoor River south & $\begin{array}{r}1,000 \\
\$ 2.50\end{array}$ & $\begin{array}{l}10,000 \\
\$ 22.00\end{array}$ \\
\hline Privet, Amoor River, north ................ No. 1 & 5.00 & 40.00 \\
\hline Trivet, Amoor River, north ................... No. 2 & ..... 3.50 & 30.00 \\
\hline Privet ,California ................. 1 & ... 2.00 & 17.50 \\
\hline l'rivet .California .................. . . 2 & 1.25 & 10.00 \\
\hline Privet, Ilota & ...... 4,50 & 40.00 \\
\hline 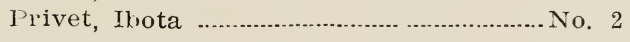 & ... 2.25 & 20.00 \\
\hline r'rivet, Tulgar'is ........ & 4.00 & 35.00 \\
\hline 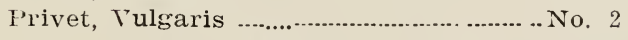 & ... 3.00 & 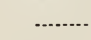 \\
\hline Privet, Vulgaris Marginata Al:pentea.... No. 1 & ..... 10.00 & ......... \\
\hline 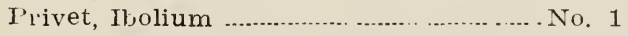 & ... 10.00 & ........ \\
\hline 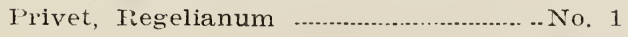 & …. 5.00 & \\
\hline 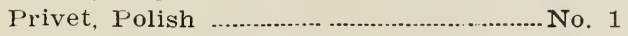 & 10.00 & \\
\hline Privet, California Japoniem .................... No. 1 & 10.00 & \\
\hline Privet, Macrophyllum .................. & 10.00 & \\
\hline \multirow[t]{3}{*}{$\begin{array}{c}\text { Privet, rhinensis Pendulifolium .......... No. } 1 \\
\text { Spireas }\end{array}$} & ...... 15.00 & 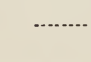 \\
\hline & Per & Per \\
\hline & 100 & 1,000 \\
\hline Anthony Waterer & $\$ 2.00$ & $\$ 15.00$ \\
\hline ........ No. 1 & 75 & 4.00 \\
\hline No. 1 & ........................... & 3.50 \\
\hline No. 1 & (n) & 5.00 \\
\hline 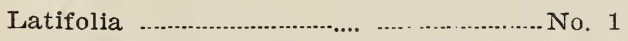 & .60 & 4.00 \\
\hline Oppulifolia & .60 & 3.50 \\
\hline 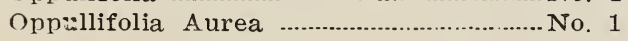 & 1.50 & 5.00 \\
\hline 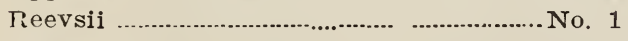 & . & 3.50 \\
\hline 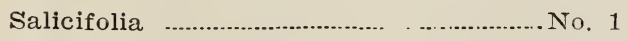 & .75 & 4.00 \\
\hline 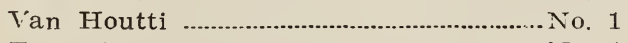 & .60 & 4.00 \\
\hline Tomentosum & & 4.00 \\
\hline
\end{tabular}


Poplars Carolina ..................................... No.

\begin{tabular}{|c|c|c|}
\hline 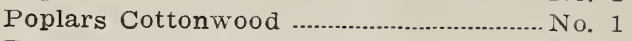 & .60 & $\begin{array}{l}3.00 \\
3.00\end{array}$ \\
\hline 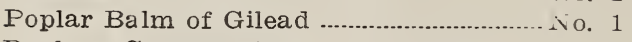 & .75 & 5.00 \\
\hline oplars Canadensis ...................................... No. 1 & 1.00 & 6.00 \\
\hline Foplars Lombardy .................................. 1 & .50 & 3.50 \\
\hline 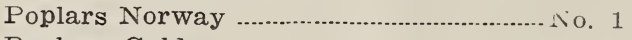 & .60 & 1.00 \\
\hline Pcplars Golden ............ No. 1 & .75 & .00 \\
\hline Poplars Silver Leaf .................... No. 1 & .50 & .50 \\
\hline Poplars Simoni (Chinese) ............................ 1 & .75 & .00 \\
\hline Willow Babylonian ................................... No. 1 & .50 & 3.50 \\
\hline Willow Britzensis ........................................... 1 & .50 & .50 \\
\hline Willow Alba Frfagilis ..................... No. 1 & .75 & 5.00 \\
\hline Willow Discolor (Pussy Willow) ........ No. 1 & .50 & .00 \\
\hline Willow Dolorosa (Thurlaws) ................ No. 1 & .50 & 4.00 \\
\hline 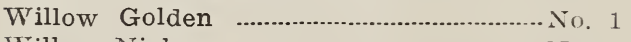 & .75 & 5.00 \\
\hline Willow Niobe ................. No. 1 & .50 & 4.00 \\
\hline 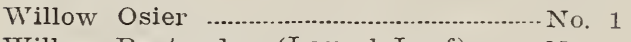 & .50 & 4.00 \\
\hline Willow Pentandra (Laurel Leaf) ......... No. 1 & .75 & 5.00 \\
\hline Willow Rosamarinifolia (Rosemary) .... No. 1 & .75 & 5.00 \\
\hline 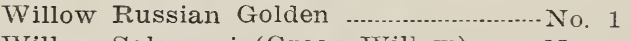 & .50 & 4.00 \\
\hline Willow Salamoni (Green Willow) .......... No. 1 & .75 & 5.00 \\
\hline 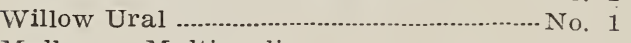 & .50 & 4.00 \\
\hline Mulberry Multicaulis .................. No. 1 & .75 & 5.00 \\
\hline - & & \\
\hline
\end{tabular}

-igs, Texas Wonder, said to be the

most wonderful Fig in cultivation.

8 1-2 inches around, and a dozen

will make a gallon ....................... 1 .................. 2.00

Rose, Crimson Rambler .......................... No. 1

Rose, Cherokee White .............................. No. 1

Rose, Dorothy Perkins Pink ................... No. 1

Fose ,Excelsa ............................................. No. 1

Rose, Gardenia .............................................. 1

Fose, Madam Plantier ................................ 1

Rose, Rugosa Type ................................... No. 1

Rose ,Seren Sisters ................................... No. 1

Rose ,Wichurianna Type ......................... No. 1

Rose, Blanda ............................................... No. 1

Rose ,Carolina ........................................... 1

Rose, Pauls Scarlet Climber ....................... 1

Rose, Climbing American eBauty ........ ? . 1

Rose, Silver Moon …..................................... 1

Rose, Queen of Prairie .......................... 1 ......................... 1.00

Rose Cinnamom Budding

.................................... No. 1 ........................ $\quad .75$

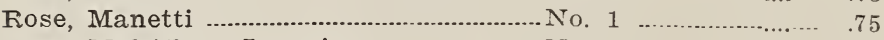

Rose ,Multiflora Japonica ......................... No. 1 ...................... .75

Rose, Wax ................................................ 1 ...................... 75

HARDY FLOWERING SHRUBS

$\begin{array}{ccl}\text { Per } & \text { Per } & \text { Per } \\ 100 & 1.000 & 10.000 \\ \$ 2.00 & \$ 15.00 & \$ \ldots . .\end{array}$

Abelia Grandiflora

Althea Amplissimus, Ardens, Bicolor

Althea Boule de Feu, Ellegantissima

Althea Joan of Arc, Lady Stanley, Lucy.

Princess Louise, Peohiflora, Totus Albus .... $.50 \quad 4.00 \quad 35.00$

Broussenetia Papyifera (Paper Mulberry) ....... . . 55050040

Putterfly Bush (Buddleia Magnifica

Variabillis) 
Cornus Alternifolia ................................................ .50

Cornus Lutia (Golden) ............................................. 1.00

4.00

30.00

Cornus Red Twig ................................................... $\quad .50$

Cornus Siberica ..................................................... 1.00

Cornus Stolonifera ..................................................... $\quad .75$

Euonymus Japonica ............................................... 1.50

Fuonymus Aurea (Golden Variegated) ................ 2.00

Euonymus Sieboldii ..................................................... 1.25

Deutizia Candidissima, Crenata Rosea fl, pl.)

Fortuni, Pride of Rochester ....................... $\quad .50$

Forsythia Intermedia, Viridissima ..................... $\quad .50$

Forsythia Intermedia Aurea, (Golden Var.)....... 1.50

Forsythia Suspensa ............................................. 1.00

Forsythia Variegated Argentea (Silver Leaf) .... 1.50

Forsythia Fortuni ................................................ 1.00

Honeysuckle, Hall's Japan ................................... .50

Hydrangea Arborescens (vrild) .............................. 1.00

Hydrangea Paniculata Grandiflora ....................... 1.50

Hypericum Prolificum ............................................ 1.00

Jasemine Nudiflora (Yellow Jasemine) ................ 1.50

Lonicera (Bush Honeysuchle) Belle Albida,)

Fragrantissima, Grand, Rosea, Morrowi)

Tartarian Pink, Tartarian White ............... .60

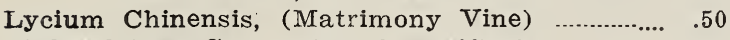

Philadelphus, Coronarius, Grandifloria)

Trodona Grandifloria fl. pl) ..................... $\quad .50$

Pomegranite Flowering double red ....................... 1.00

Sambucus Canadensis ............................................ $\quad .75$

Weigelia Amabillis, Desboisi, Gigantiflora,

Hendersoni, Stelzneri .................................. .75

Wiegelia Rosea ..................................................... 1.25

Symphoricarpos Acutis ........................................ 1.00

Symphoricarpos Racemosus .............................. 1.00

Symphoricarpos Vulgaris ........................................ $\quad .50$

Stephandra Flexuosus ............................................. 1.00

Tamarix Gallacia, Odessanna ............................ 1.00

Tamarix Hispida Aestivalis .................................. 1.25

Nursery Grown Shade Trees-s, seedlings; c, cuttings; tr, transplanted

Per Per

$100 \quad 1000$

Ailanthus glandulosus (s)

$4.00 \quad 30.00$

6.00

5.00

12.50

15.00

10.00

$\begin{array}{rr}4.00 & 35.00 \\ 4.00 & 35.00 \\ 6.00 & \ldots \ldots . . \\ 6.00 & \ldots \ldots . . . \\ 6.00 & \ldots \ldots . . \\ 4.00 & \ldots \ldots . . \\ 3.50 & 30.00 \\ 6.00 & 50.00 \\ 10.00 & \ldots \ldots . . \\ 6.00 & 50.00 \\ 10.00 & \ldots \ldots . .\end{array}$

$5.00 \quad 40.00$

3.50

$3.50 \quad 30.00$

$5.00 \quad 40.00$

4.00

$6.00 \quad 50.00$

$8.00 \quad 60.00$

6.00

6.00

3.50

25.00

800

$6.00 \quad 50.00$

$\$ 1.00 \$ 7.00$

6 to 12 inches ........................................................ $\$ 1.00$

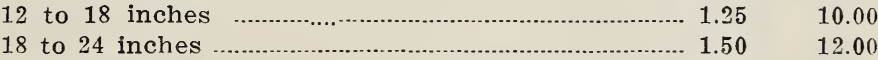

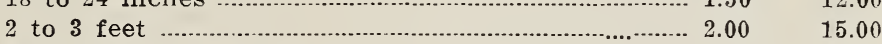

3 to 4 feet ................................................................... 4.00

Aeer Dasycarpum (Silver Maple)

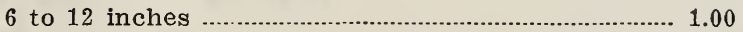

12 to 18 inches ............................................................. 1.25

18 to 24 inches ................................................................ 1.50

2 to 3 feet ................................................................. 2.00

Albizzia Julibrissin (Mimosa Tree)

4 to 6 inches ....................................................... 5.00

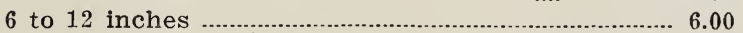

12 to 18 inches ............................................................... 8.00

18 to 24 inches .................................................................. 100

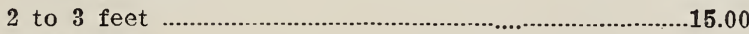

Catalpa Speciosa

6 to 12 inches ............................................................... $100 \quad 4.00$

12 to 18 inches ........................................................... $1.00 \quad 5.00$

18 to 24 inches ............................................................ $1.00 \quad 7.00$

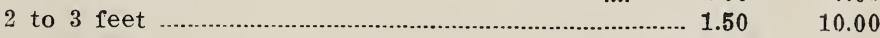

3 to 4 feet ..................................................................... $3.00 \quad 25.00$

4 to 5 feet .................................................................... $5.00 \quad 40.00$ 
Laburnam Vulgare (Golden chain) (s)

6 to 12 inches

12 to 18 inches ................................................... 3.50

18 to 24 inches .............................................................. 4.00

Mulberry Russian (s)

6 to 12 inches ............................................................. 1.00

12 to 18 inches ................................................................ 1.25

18 to 24 inches ....................................................... 1.50

7.50

2 to 3 feet ............................................................... 2.00

Poplar Lombardy, Whips (c)

6 to 12 inches

30.00

12 to 18 inches

12.00

15.00

18 to 24 inches

2 to 3 feet

25.00

3 to 4 feet ................................................................. $4.00 \quad 35.00$

Foplar Silver Leaf, Whips (c)

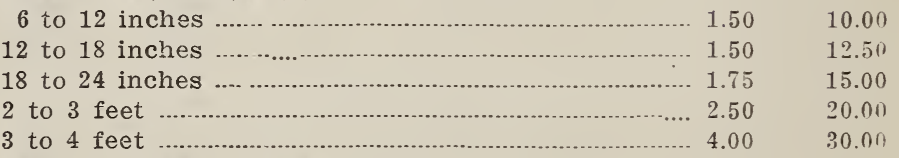

Poplar Balm of Gilead Whips (c)

6 to 12 inches ............................................................. $2.00 \quad 15.00$

1 to 2 feet .................................................................. $3.00 \quad 25.00$

2 to 3 feet ................................................................... $4.00 \quad 35.00$

Sycamore, American (s)

6 to 12 inches .............. 1.00

12 to 18 inches ............................................................ 1.50

18 to 24 inches

\section{DECIDUOUS SHRUBS}

Nursery Grown-s, seedlings; c, cuttings; tr, transplanted

Althea Rosea, mixed colors (s)

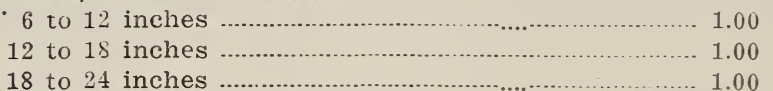

Boule de Feu, doutle red (c)

Althea Boule de Feu,
6 to 12 inches

12 to 18 inches

Albizzia Julibrissin (Mimosa Tree) (s)

4 to 6 inches

6 to 12 inches

12 to 18 inches

18 to 24 inches

Amorpha Fruticosa (False Indigo (s)

6 to 12 inches

0.00

12 to 18 inches

18 to 24 inches

Cydonia Japonica (s)

6 to 12 inches

12 to 18 inches

18 to 24 inches

Cercis Canadensis (Red Bud) (s)

12 to 18 inches

18 to 24 inches

2 to 3 feet

Eleagnus Augustifolia (s)

6 to 12 inches 
Forsythia Intermedia (c)

6 to 12 inches .................................................................... $3.00 \quad 20.00$

12 to 18 inches ....................................................... $2.50 \quad 30.00$

Forsythia Viridissima (c)

6 to 12 inches ................................................................. $3.00 \quad 20.00$

12 to 18 inches ................................................................. $3.50 \quad 30.00$

Hydrangea Arborescens (wild) (tr)

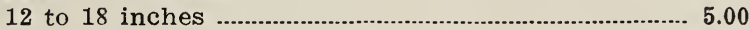

18 to 24 inches ................................................................ 7.00

Privet South (s)

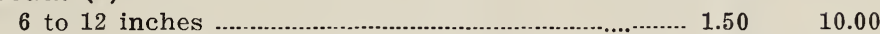

12 to 18 inches ................................................................ $2.50 \quad 20.00$

18 to 24 inches ................................................................ $5.00 \quad 35.00$

2 to 3 feet ................................................................... $8.00 \quad 60.00$

Privet California (c)

6 to 12 inches ............................................................. $1.00 \quad 8.00$

Privet Amoor River Nortin (c)

6 to 12 inches ............................................................. $2.50 \quad 20.00$

Rhus Glabra (s)

6 to 12 inches ................................................................ $1.25 \quad 10.00$

12 to 18 inches ................................................................. $1.50 \quad 12.50$

188 to 24 inches ........................................................... $2.00 \quad 17.50$

2 to 3 ieet ................................................................. $3.00 \quad 25.00$

Rhus Typhina (s)

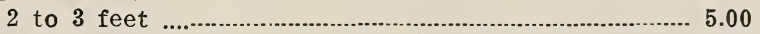

Spirea Van Houtti (c)

6 to 12 inches .............................................................. $3.00 \quad 25.00$

12 to 18 inches ........................................................... $5.00 \quad 35.00$

Spirea Reevsi (c)

6 to 12 inches ................................................................... $3.00 \quad 25.00$

12 to 18 inches ............................................................. $5.00 \quad 35.00$

Symphoracarpos Vulgaris (c)

6 to 12 inches ......................................................... 2.00

COLLECTED SEEDLINGS-TREES AND SHRUBS

Orders should be placed for this stock as early as possible, as it is all pulled from the woods on wet cloudy days.

Aronia Niger, Black Chokeberry

6 to 12 inches .............................................................. \$2.00 $\$ 10.00$

1 to 2 feet ............................................................ $2.50 \quad 15.00$

2 to 3 feet ..................................................................... $4.00 \quad 25.00$

Acer Saccharum, Sugar Maple

6 to 12 inches .......................................................... 1.00
1 to 2 feet .................................................................. 1.25

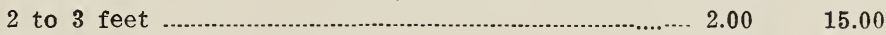

3 to 4 feet ...................................................................... $3.50 \quad 30.00$

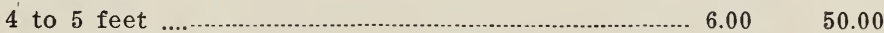

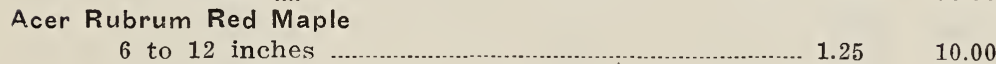

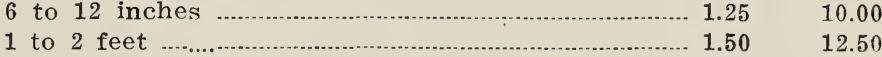

2 to 3 feet .................................................................. $2.00 \quad 15.00$

3 to 4 feet .................................................................... $3.50 \quad 25.00$

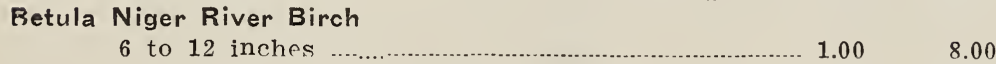

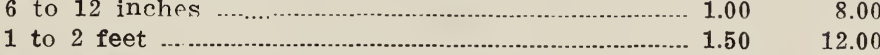

2 to 3 feet ................................................................. $2.00 \quad 15.00$

3 to 4 feet .......................................................................... $4.00 \quad 25.00$

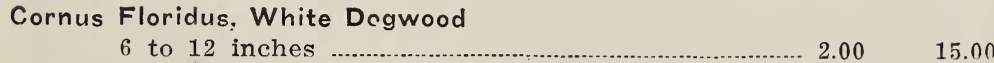

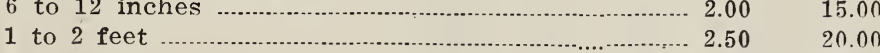

2 to 3 feet ............................................................... $4.00 \quad 35.00$

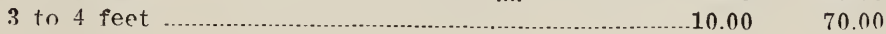


Celastrus Scandens, Bittersweet

6 to 12 inches ......................................................... $2.00 \quad 10.00$

1 to 2 feet .................................................................. $3.00 \quad 20.00$

2 to 3 feet ..................................................................... $4.00 \quad 30.00$

Hydrangea Arborescens, (Wild)

1 to 2 feet stems ....................................................... $2.00 \quad 12.00$

2 to 3 feet stems .......................................................... $3.00 \quad 20.00$

3 to 4 feet stems ........................................................ $4.00 \quad 30.00$

Hypericum Prolificum

6 to 12 inches ............................................................. 2.00 12.00

1 to 2 feet ................................................................... $2.50 \quad 20.06$

2 to 3 feet ................................................................ $5.00 \quad 40.00$

Huckleberry, Dwarf, Summer

6 to 12 inches ..................................................... $1.25 \quad 10.00$

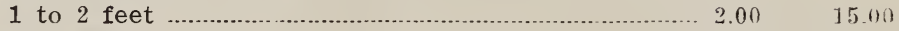

Lirodendron, Tulip Poplar

6 to 12 inches ....................................................... $1.25 \quad 10.00$

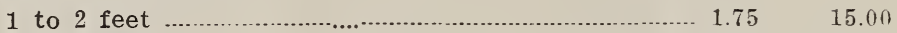

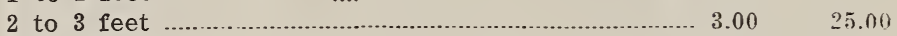

3 to 4 feet ............................................................. $5.00 \quad 40.00$

Rhamnus Carolinianna, Buckthorn

6 to 12 inches .......................................................... $1.00 \quad 8.00$

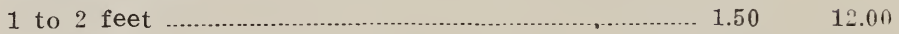

2 to 3 feet ................................................................. $2.00 \quad 15.00$

Rhus Aromatica, Suckers, cut back

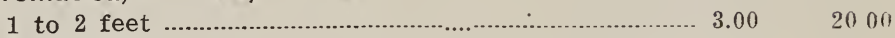

2 to 3 feet ........................................................................ $4.00 \quad 30.00$

Sambucus Canadensis, Common Elder

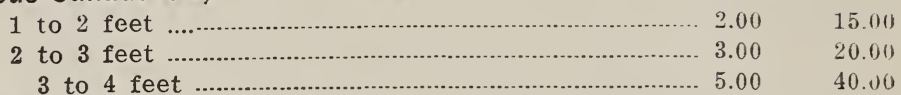

Viburnam Acerfolium, Arrowwood

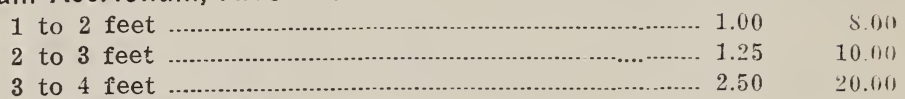

Quercus, Nigra, Black Oak

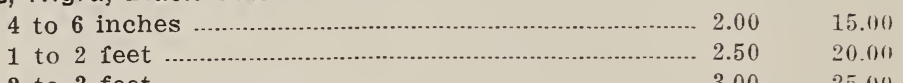

2 to 3 feet .................................................................. $3.00 \quad 25.00$

Quercus, Phellos, Willow Oak

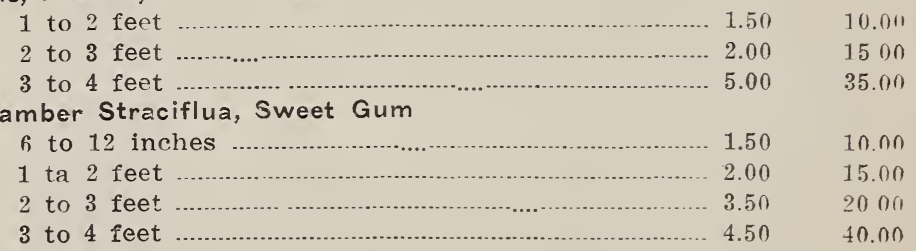

Azalea, Wild

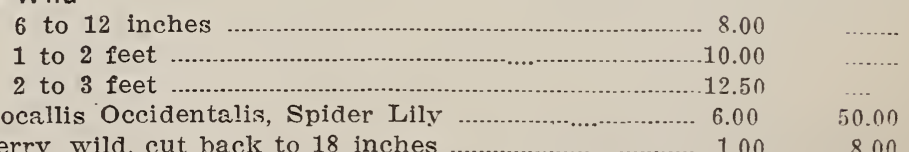

Hymenocallis Occidentalis, Spider Lily …......................... 6.00 50.00

VINES. Collected

Ampelopsis Quinquefolia, Virginia Creeper $100 \quad 1000$

Bignonia Radicans, Trumpet Flower ..................................................

Honeysuckle, Hall's Japan, small ........................................ $1.00 \quad 8.0 n$

Honeysuckle, Hall's Japan, medium .................................. 1.50 12.50

Kudzu, nursery grown, 1 year ................................................ 
TREE AND SHRUB SEED

Orders are accepted for seed subject to crop conditions, and with the understanding that no warranty either expressed or implied is given as to germination, or as to genuineess.

$\begin{array}{ll}\text { Acer Dasycarpum, Silver Leaf Maple..... May } & \text { Mate } \\ \text { Ready }\end{array}$

Per Per

Acer Negundo, Ash Leaf Maple ............. Oct.

Acer Pseudo Platanus ,Sycamoe ..............Dec.

Acer Rubrum, Red ,or Swamp Maple .... May

Acer Saccharum ,Sugar, or Rock Maple Sept.

Acer Platanoides, Norway Maple .........July

Ailanthus glandulosus, eHaven tree .... Nov.

Albizzia Julibrissin, Mimosa tree ......... Nov.

Ampelopsis Virginica Va. Creeper ......... Dec.

Amorpha Fruticosa, False Indigo ......... Sept.

Amelanchier botryapium, Juneberry .... July

Aralia Spinosa, Angelica tree ................ Dec.

Asparagus: Barr's Mammoth, Conov-

er's Collossal, Giant argenteuil,

Palmetto .............................................. Nov.

oz. $\quad$ lb.

$\$ .10 \$ .60$

$.15 \quad .75$

$.15 \quad 1.00$

$.15 \quad 1.00$

$.15 \quad 1.00$

$.20 \quad 2.00$

$.15 \quad .60$

$.25 \quad 2.00$

$.25 \quad 1.75$

$.10 \quad .75$

$.50 \quad 5.00$

$.30 \quad 2.50$

Asparagus, Washington Rust Proof .... Nov.

Berberis thunbergii, Japan Barberry ....Dec. atalpa Speciosa (Western) …............. Dec.

Calicarpa Americanna, Beauty Bush.... Nov.

Calycanthus Floridus .............................. Dec.

Carya, Alba, Shell bark hickory ......... Nov.

Carya, Sulcata, Western Shell bark

hickory .............................................. Nov.

Cephalanthus Occidentalis, Buttonbush Nov.

Cercis canadensis, Red bud .................... Nov.

Citrus Trifoliata, Hardy orange ............. Nov.

Citrus Auranticum, Sour orange ............. Dec.

Cornus floridus, White flowering dog-

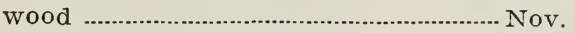

Corylus Americanna, Hazelnut ................ Nov.

Crataegus cordata .................................... Dec.

Crataegus Crus-Galli, Cocksput thorne Dec.

Crataegus Coccinea, Thicket thorn ......... Dec.

Cydonia Japonica, Japan Quince ........ Dec.

Diospyros Virginica ,Am. Persimmon.. Dec.

Exochorda Grand, Pearl Bush ............... Nov.

Fraxinus Americana, White Ash ........ Nov.

Fraxinus Lanceola (Viridis) Green Ash Nov.

Halesia Diptera, Silver bell .................... Nov.

Falesia, Tetrantera, Great silver bell.. Nov.

Hibiscus Syriacus, Althea Nov. ............ Nov.

Hydrangea Quercifolia (Oak leaf) ......... Nov.

Hamamelis Virginica, Witch Hazel .... Nov.

Hypericum Prolificum Pods ................ Nov.

Ilex Opaca, Am. Holly ............................. Dec.

Juniperus Virginianna, Red Cadar .......Dec.

Iigustrum Amurense, A. River Privet

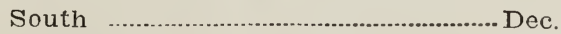

Ligustrum Ibota, Ibota Privet ................. Dec.

Lisustrum Janonicum, Janan Privet .... Dec.

Ligustrum Lucidum, Glossy Privet ....Dec.

Ligustrum Sinense, Chinese Privet .... Dec.

Liquidamber Styraciflua, Sweet fum.... Dec.

Laurocerasus Carolinianna, Wild 
Magnolia Tripetala .................................. Oct.

Nyssa Multiflora, Black Gum ................ Oct.

Nyssa Uniflora, Tupelo Gum ............... Oct.

Pawlonia Tomentosa, (Imperialis) .... Nov.

Pyrus Callerianna, Japan Pear ........... January $\quad$.

Fyrus Ussuriensis, Japan Pear …....... January $\quad 1.00 \quad 8.00$

I'runus Serotina, Wild Cherry ............Juiy

Rhamnus Carolinianna, Buckthorn .... Oct. $\quad .20 \quad 1.50$

Rhus Aromatica, (canadense) Sweet

Sumach ................................................. Nor.. $.0 \quad 1.011$

Rhus Cotinus, Purple fringe, smoke

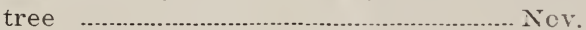

Rhus Copalina, Black Sumac ................ Nur.

Rhus Glabra, Smooth Sumac ................ Nor.

Rhus Typhina Stag Horn Sumac ........ Nот.

Robinia Pseudacacia, Black Locust .... Dec.

Spirea Callosa Rosea ............................... Nov.

Styrax Japonica ........................................ Nov.

Quercus Alba, White Oak ...................... Nov.

Cuercus Macrocarpa, Burr Oak ............ Nov.

Quercus Lyrata, Overcup Oak ................ Nov.

Quercus Phellos Willow Oak .................... Nov.

Quercus Rubra, Red Oals ........................ Nov.

Note-If oak are wanted in quantity wite us fo: mices.

Ulmus Americanna, White Elm ........... April

Viburnam Dentatum, Arrowwood ........ Oct.

Tiburnam Prunifolum, Black Haw .... Nox.

Juglans Cinerå, Butternut ........................ Nov.

Juglans Nigra, Black Walnut ............... Dec.

Juglans Regia, English Walnut ........... Dec.

Juglans Cordiformis, Japan Walnut .... Dec.

Juglans Sieboldi, Japan Walnut ......... Dec.

Note-Will collect any seed in season that grows in our section.

Note-If you have no treceived our price list of Roses. Privets an!l Hardy Flowering Shrubs, ask us for it.

ROSEBANK NURSERY CO., Inc.,

Huntsrille, Alahama.

\section{NOTICE}

Huntsville, Ala., is the largest nursery center in the South.

The soil and climate are ideal for propagating and srowing.

Many items can be produced for one-half the cost in the Fast and North.

Many items can be grown from Hardwood cuttings, PLANTED in the "OPEN FIELD" in the fall.

Huntsville, with a population of 23,000 including suburbs, is as pleasant a small city to live in as can be found anywhere.

It costs less to grow ornamentals in Iuntsville than elsewhere.

Our soil is the most iertile in the state.

Our climate is ahead of any of the southeastern states for "yea! round."

Come to Huntsville and grow old gracefully.

Land and labor are cheap and profits certain in the nunsery business at Huntsville.

Advantageous soil and climate enable us to grow a better plant in two years for less expense than three rears north or east. 